\title{
A Note on Convergence of a Sequence and its Applications to Geometry of Banach Spaces
}

\author{
Hemant Kumar Pathak \\ School of Studies in Mathematics, Pandit Ravishankar Shukla University, Raipur, India \\ E-mail: hkpathak05@gmail.com \\ Received January 9, 2011; revised April 22, 2011; accepted April 30, 2011
}

\begin{abstract}
The purpose of this note is to point out several obscure places in the results of Ahmed and Zeyada [J. Math. Anal. Appl. 274 (2002) 458-465]. In order to rectify and improve the results of Ahmed and Zeyada, we introduce the concepts of locally quasi-nonexpansive, biased quasi-nonexpansive and conditionally biased quasi-nonexpansive of a mapping w.r.t. a sequence in metric spaces. In the sequel, we establish some theorems on convergence of a sequence in complete metric spaces. As consequences of our main result, we obtain some results of Ghosh and Debnath [J. Math. Anal. Appl. 207 (1997) 96-103], Kirk [Ann. Univ. Mariae Curie-Sklodowska Sec. A LI.2, 15 (1997) 167-178] and Petryshyn and Williamson [J. Math. Anal. Appl. 43 (1973) 459-497]. Some applications of our main results to geometry of Banach spaces are also discussed.
\end{abstract}

Keywords: Locally Quasi-Nonexpansive, Biased Quasi-Nonexpansive, Conditionally Biased

Quasi-Nonexpansive, Drop, Super Drop

\section{Introduction}

In the last four decades of the last century, there have been a multitude of results on fixed points of nonexpansive and quasi-nonexpansive mappings in Banach spaces (e.g., [5-7, 9-11]).

Our aim in this note is to point out several obscure places in the results of Ahmed and Zeyada [J. Math. Anal. Appl. 274 (2002) 458-465]. In order to rectify and improve the results of Ahmed and Zeyada, we introduce the concepts of locally quasi-nonexpansive, biased quasinonexpansive and conditionally biased quasi-nonexpansive of a mapping w.r.t. a sequence in metric spaces.

Let $X$ be a metric space and $D$ a nonempty subset of $X$. Let $T$ be a mapping of $D$ into $X$ and let $F(T)$ be the set of all fixed points of $T$. For a given $x_{0} \in D$, the sequence of iterate $\left\{x_{n}\right\}$ is determined by

$$
x_{n}=T\left(x_{n-1}\right)=T^{n}\left(x_{0}\right), n=1,2,3 \cdots
$$

Let $X$ be a normed space, $\lambda \in(0,1)$ and $\mu \in(0,1)$, the sequence of iterates $\left\{x_{n}\right\}$ are defined by

$$
\begin{aligned}
& x_{n}=T_{\lambda}\left(x_{n-1}\right)=T_{\lambda}^{n}\left(x_{0}\right), \\
& T_{\lambda}=\lambda I+(1-\lambda) T, n=1,2,3 \cdots
\end{aligned}
$$

$$
\begin{aligned}
& x_{n}=T_{\lambda, \mu}\left(x_{n-1}\right)=T_{\lambda, \mu}^{n}\left(x_{0}\right), \\
& T_{\lambda, \mu}=(1-\lambda) I+\lambda T[(1-\mu) I+\mu T], \\
& n=1,2,3 \ldots .
\end{aligned}
$$

The iteration scheme (I) is called Teoplitz iteration and the iteration scheme (II) was introduced by Mann [12] while the iteration scheme (III) was introduced by Ishikawa [9].

The concept of quasi-nonexpansive mapping was initiated by Tricomi in 1941 for real functions. It was further studied by Diaz and Metcalf [5] and Doston [6,7] for mappings in Banach spaces. Recently, this concept was given by Kirk [10] in metric spaces as follows:

Definition 1.1. The mapping $T$ is said to be quasinonexpansive if for each $x \in D$ and for every $p \in F(T)$, $d(T(x), p) \leq d(x, p)$. A mapping $T$ is conditionally quasi-nonexpansive if it is quasi-nonexpansive whenever $F(T) \neq \varnothing$.

We now introduce the following definition:

Definition 1.2. The mapping $T$ is said to be locally quasi-nonexpansive at $p \in F(T)$ if for each $x \in D$, $d(T(x), p) \leq d(x, p)$.

Obviously, quasi-nonexpansive locally quasi-nonexpansive at each $p \in F(T)$ but the reverse implication 
may not be true. To this end, we observe the following example.

Example 1.1. Let $X=[0,1)$ and $D=\left[0, \frac{3}{4}\right)$ be endowed with the Euclidean metric d. Define the mapping $T: D \rightarrow X$ by $T(x)=\frac{3}{2} x^{2}$ for each $x \in D$. Then we observe that $F(T)=\left\{0, \frac{2}{3}\right\}$, for all $x \in D$ and $p=0$ $\in F(T)$, we have that

$$
d(T(x), p)=\left|\frac{3}{2} x^{2}-0\right| \leq|x-0|=d(x, p),
$$

i.e., $T$ is locally quasi-nonexpansive at $p=0 \in F(T)$. However, one can easily see that $T$ is not locally quasinonexpansive at $p=\frac{2}{3} \in F(T)$. Indeed, for all $x \in\left(0, \frac{2}{3}\right)$ and $p=\frac{2}{3} \in F(T)$ we have

$$
d(T(x), p)=\left|\frac{3}{2} x^{2}-\frac{2}{3}\right|>\left|x-\frac{2}{3}\right|=d(x, p) .
$$

Hence we conclude that $T$ is not quasi-nonexpansive, although it is locally quasi-nonexpansive at $p=0 \in F(T)$.

The concept of asymptotic regularity was formally introduced by Browder and Petryshyn [3] for mappings in Hilbert spaces. Recently, it was defined by Kirk [11] in metric spaces as follows:

Definition 1.3. The mapping $T$ is said to be asymptotically regular if $\lim _{n \rightarrow \infty} d\left(T^{n}(x), T^{n+1}(x)\right)=0$ for each $x \in D$.

\section{Main Results}

Let $\mathbf{N}$ denote the set of all positive integers and $\omega$ $=\mathbf{N} \cup\{0\} \quad$ Ahmed and Zeyada [1] introduce-ed the following:

Definition 2.1. The mapping $T$ is said to be quasinonexpansive w.r.t. a sequence $\left\{x_{n}\right\}$ if for all $n \in \omega$ and for each $p \in F(T), d\left(x_{n+1}, p\right) \leq d\left(x_{n}, p\right)$.

The following lemma was quoted by Ahmed and Zeyada [1] without proof.

Lemma A. If $T$ is quasi-nonexpansive, then $T$ is quasi-nonexpansive w.r.t. a sequence $\left\{T^{n} x_{0}\right\}$ (respectively, $\left\{T_{\lambda}^{n} x_{0}\right\},\left\{T_{\lambda, \mu}^{n} x_{0}\right\}$ ) for each $x_{0} \in D$.

Remark 2.1. We notice that the above lemma is valid if $\left\{T^{n} x_{0}\right\} \in D$ for each $n \in \omega$ and a given $x_{0} \in D$ (or $D$ is $T$-invarient). So the correct version of Lemma A should be read as follows:

Lemma 2.1. If $T$ is quasi-nonexpansive and for a given $x_{0} \in D$ and each $n \in \omega,\left\{T^{n} x_{0}\right\} \in D$, then $T$ is quasi-nonexpansive w.r.t. a sequence $\left\{T^{n} x_{0}\right\}$ (respectively, $\left\{T_{\lambda}^{n} x_{0}\right\},\left\{T_{\lambda, \mu}^{n} x_{0}\right\}$ ) for each $x_{0} \in D$.

Further, they claimed that the reverse implication in Lemma A may not be true in their Example 2.1. We again notice that there are several obscure places in this example. We now quote Example 2.1 of Ahmed and Zeyada [1] in the following:

Example A. Let $X=[0,1)$ and $D=\left[0, \frac{4}{5}\right)$ be endowed with the Euclidean metric d We define the mapping $T: D \rightarrow X$ by $T(x)=2 x^{2}$ for each $x \in D$. For a given $x_{0}=\frac{1}{4} \in D$ we have

$$
\begin{aligned}
d\left(T^{n+1}\left(x_{0}\right), p\right) & =\left|\left(\frac{1}{2}\right)^{2^{n+1}+1}-0\right| \leq\left|\left(\frac{1}{2}\right)^{2^{n}+1}-0\right| \\
& =d\left(T^{n}\left(x_{0}\right), p\right)
\end{aligned}
$$

where $T^{n}(1 / 4)=(1 / 2)^{2^{n}+1} \in D \forall n \in \mathbf{N} \cup\{0\}$ and $\mathrm{F}(\mathrm{T})$ $=\{0\}$, i.e., $T$ is quasi-nonexpansive w.r.t. a sequence $T^{n}(1 / 4)$ Furthermore, the map $T$ is quasi-nonexpansive w.r.t. a sequence $\left\{T_{1 / 2}^{n}(1 / 2)\right\}$ and $\left\{T_{1 / 2,1 / 2}^{n}(1 / 2)\right\}$. They found that $T$ is neither conditionally quasi-nonexpansive nor quasi-nonexpansive, for $x=\frac{3}{4} \in D$ and $p=0 \in F(T), d(3 / 4,0)>d(3 / 4,0)$ and $D$ is not closed.

Remark 2.2. We notice that the following claims made in Example A were false:

1) $T: D \rightarrow X$ is a mapping. In fact,

$$
T(D)=\left[0, \frac{32}{25}\right) \supset[0,1)=X \text {. }
$$

2) $\mathrm{F}(\mathrm{T})=\{0\}$, In fact, $F(T)=\left\{0, \frac{1}{2}\right\}$.

3) $\mathrm{T}$ is quasi-nonexpansive w.r.t. a sequence $\left\{T^{n}\right.$ $(1 / 4)\}$.

4) $\mathrm{T}$ is quasi-nonexpansive w.r.t. a sequence $\left\{T_{1 / 2}^{n}(1 / 2)\right\}$ and $\left\{T_{1 / 2,1 / 2}^{n}(1 / 2)\right\}$.

However, (i) can be rectified by taking $X$ as $\left[0, \frac{32}{25}\right)$ or any superset of $\left[0, \frac{32}{25}\right)$ in $[0, \infty)$ Even if this correction is made we find that the remaining statements 2) - 4) will remain false. Consequently, the claim of Ahmed and Zeyada [1] that the reverse implication in Lemma 2.1 may not be true seems false.

We now introduce the following definition.

Definition 2.2. The mapping $T$ is said to be locally quasi-nonexpansive at $p \in F(T)$ w.r.t. a sequence $\left\{x_{n}\right\}$ 
if for all $n \in \omega, d\left(x_{n+1}, p\right) \leq d\left(x_{n}, p\right)$.

Obviously, locally quasi-nonexpansiveness at $p \in$ $F(T) \Rightarrow$ locally quasi-nonexpansiveness at $p \in F(T)$ w.r.t. a sequence $\left\{x_{n}\right\}$.

We now state the following lemma without proof.

Lemma 2.2. If $T$ is quasi-nonexpansive w.r.t. a sequence $\left\{x_{n}\right\}$ then $T$ is locally quasi-nonexpansive at each $p \in F(T)$ w.r.t. the sequence $\left\{x_{n}\right\}$.

The reverse implication in Lemma 2.2 may not be true as shown in the following example:

Example 2.1. Let $X=[0,1)$ and $D=\left[0, \frac{2}{3}\right)$ be endowed with the Euclidean metric d Define the map- ping $T: D \rightarrow X$ by $T(x)=2 x^{2}$ for each $x \in D$ Then we observe that $F(T)=\left\{0, \frac{1}{2}\right\}$. For a given $x_{0}=\frac{1}{4} \in D$ and $p=0 \in F(T)$ we have that

$$
\begin{aligned}
& d\left(T^{n+1}\left(x_{0}\right), p\right)=\left|\left(\frac{1}{2}\right)^{2^{n+1}+1}-0\right|<\left|\left(\frac{1}{2}\right)^{2^{n}+1}-0\right| \\
& =d\left(T^{n}\left(x_{0}\right), p\right)
\end{aligned}
$$

where $T^{n}\left(\frac{1}{4}\right)=\left(\frac{1}{2}\right)^{2^{n}+1} \in D$ i.e., $T$ is locally quasinonexpansive at $p=0 \in F(T)$ w.r.t. a sequence $\left\{T^{n}\left(\frac{1}{4}\right)\right\}$ However, one can easily see that $T$ is not locally quasi-nonexpansive at $p=\frac{1}{2} \in F(T)$ w.r.t. the sequence $\left\{T^{n}\left(\frac{1}{4}\right)\right\}$. Indeed, we have

$$
\begin{aligned}
d\left(T^{n+1}\left(x_{0}\right), p\right) & =\left|\left(\frac{1}{2}\right)^{2^{n+1}+1}-\frac{1}{2}\right|>\left|\left(\frac{1}{2}\right)^{2^{n+1}+1}-\frac{1}{2}\right| \\
& =d\left(T^{n}\left(x_{0}\right), p\right)
\end{aligned}
$$

for all $n \in \omega$ Consequently, $T$ is neither quasi-nonexpansive nor quasi-nonexpansive w.r.t. the sequence $\left\{T^{n}\left(\frac{1}{4}\right)\right\}$.

We now introduce the following:

Definition 2.3. The mapping $T: D \rightarrow X$ is said to be biased quasi-nonexpansive (b.q.n) w.r.t. a sequence $\left\{x_{n}\right\} \subset X$ if for all $n \in \omega$ and at each $p \in \operatorname{cond}(F(T))$,

$$
d\left(x_{n+1}, p\right) \leq d\left(x_{n}, p\right)
$$

where

$$
\begin{aligned}
& \operatorname{cond}(F(T))=\{p \in F(T): \limsup _{n \rightarrow \infty} d\left(x_{n}, p\right) \\
&\left.\leq \liminf _{n \rightarrow \infty} d\left(x_{n}, F(T)\right)\right\}
\end{aligned}
$$

A mapping $T$ is conditionally biased quasi-nonexpansive (c.b.q.n) w.r.t. a sequence $\left\{x_{n}\right\}$ if cond $(F(T)) \neq \varnothing$.

Remark 2.3. We observe that the following implications are obvious:

(a) Conditional biased quasi-nonexpansiveness w.r.t. a sequence $\left\{x_{n}\right\} \Rightarrow$ biased quasi-nonexpansiveness w.r.t. a sequence $\left\{x_{n}\right\}$ but the reverse implication may not be true (Indeed, any mapping $T: D \rightarrow X$ for which $\operatorname{cond}(F(T)) \neq \varnothing$ is a biased quasi-nonexpansive w.r.t. a sequence $\left\{x_{n}\right\}$ but not conditionally biased quasinonexpansive w.r.t. a sequence $\left\{x_{n}\right\}$. However, under certain conditions a biased quasi-nonexpansive map w.r.t. a sequence $\left\{x_{n}\right\}$ may be a conditional biased quasinonexpansive w.r.t. a sequence $\left\{x_{n}\right\}$ (see Lemma 2.6 below).

(b) If $T$ is conditionally biased quasi-nonexpansive w.r.t. a sequence $\left\{x_{n}\right\}$ and $\operatorname{cond}(F(T))=F(T) \neq \varnothing$ then $T$ is locally quasi-nonexpansive at each $p \in F(T)$ w.r.t. a sequence $\left\{x_{n}\right\}$.

(c) If $T$ is biased quasi-nonexpansive w.r.t. a sequence $\left\{x_{n}\right\}$ and $\varnothing \neq \operatorname{cond}(F(T)) \ddot{O} F(T)$ then $T$ is locally quasi-nonexpansive at each $p \in \operatorname{cond}(F(T))$ w.r.t. a sequence $\left\{x_{n}\right\}$.

(d) Quasi-nonexpansivenes $\Rightarrow$ locally quasi-nonexpansiveness at $p \in F(T) \Rightarrow$ locally quasi-nonexpansiveness at $p \in F(T)$ w.r.t. a sequence $\left\{x_{n}\right\}$.

In Example 2.1 above, we observe that

1) for $p=0 \in F(T)$, we have

$$
\begin{aligned}
\limsup _{n \rightarrow \infty} d\left(x_{n}, p\right) & =\limsup _{n \rightarrow \infty}\left|\left(\frac{1}{2}\right)^{2^{n}+1}-0\right| \\
& =\lim _{n \rightarrow \infty}\left(\frac{1}{2}\right)^{2^{n}+1}=0
\end{aligned}
$$

2) for $p=\frac{1}{2} \in F(T)$, we have

$$
\begin{aligned}
\limsup _{n \rightarrow \infty} d\left(x_{n}, p\right) & =\limsup _{n \rightarrow \infty}\left|\left(\frac{1}{2}\right)^{2^{n}+1}-\frac{1}{2}\right| \\
& =\lim _{n \rightarrow \infty}\left|\left(\frac{1}{2}\right)^{2^{n}+1}-\frac{1}{2}\right|=\frac{1}{2}
\end{aligned}
$$

$\liminf _{n \rightarrow \infty} d\left(x_{n}, F(T)\right)=\liminf _{n \rightarrow \infty}\left(\frac{1}{2}\right)^{2^{n}+1}=\lim _{n \rightarrow \infty}\left(\frac{1}{2}\right)^{2^{n}+1}=0$ 
Here $\operatorname{cond}(F(T)) \neq\{0\}$ and in view of $(*)$ and $(* *)$, it is evident that $T$ is conditionally biased quasi-nonexpansive (c.b.q.n.) w.r.t. a sequence $\left\{T^{n}\left(\frac{1}{4}\right)\right\}$ and hence it is biased quasi-nonexpansive (b.q.n.) w.r.t. a sequence $\left\{T^{n}\left(\frac{1}{4}\right)\right\}$.

We now show in the following example that cond $(F(T))$ need not be a singleton set.

Example 2.2. Let $X=[0,2]$ and $D=[0,1) \cup(1,2]$ be endowed with the Euclidean metric d Define the mapping $T: D \rightarrow X$ by $T x=+\sqrt{x}$ for $x \in[0,1)$ $\cup(1,2)$ and $T(x)=2$ for $x=2$. Clearly, $\mathrm{F}(\mathrm{T})$ $=\{0,2\}$ Consider the sequence $\left\{x_{n}\right\} \equiv\{1\}$ in $X$ then we observe that

1) for $p=0 \in F(T)$, we have

$$
\lim _{n \rightarrow \infty} \sup d\left(x_{n}, p\right)=\limsup _{n \rightarrow \infty}|1-0|=\lim _{n \rightarrow \infty} 1=1 ;
$$

2) for $p=2 \in F(T)$, we have

$$
\limsup _{n \rightarrow \infty} d\left(x_{n}, p\right)=\limsup _{n \rightarrow \infty}|1-2|=\lim _{n \rightarrow \infty} 1=1 ;
$$

and

$$
\lim _{n \rightarrow \infty} \inf d\left(x_{n}, F(T)\right)=\lim _{n \rightarrow \infty} 1=1 .
$$

Thus we have cond $(\mathrm{F}(T))=\{0,2\}$ and it is evident that $T$ is conditionally biased quasi nonexpansive (c.b.q.n.) w.r.t. the sequence $\left\{x_{n}\right\} \equiv\{1\}$ in $X$, and hence it is biased quasi-nonexpansive (b.q.n.) w.r.t. the sequence $\left\{x_{n}\right\} \equiv\{1\}$ in $X$.

However, interested reader can check that if we consider the sequence $\left\{x_{n}\right\}$ such that $x_{n} \rightarrow 1^{+}$then cond $(\mathrm{F}(\mathrm{T}))=\{2\}$ Further, we observe that for $p=2$ $\in \operatorname{cond}(\mathrm{F}(T))$ and for all $n \in \omega$ we have

$$
d\left(x_{n+1}, p\right) \leq d\left(x_{n}, p\right)
$$

Thus, $T$ is conditionally biased quasi-nonexpansive (c.b.q.n.) w.r.t. the sequence $\left\{x_{n}\right\}$ in $X$.

On the other hand, if we consider the sequence $\left\{x_{n}\right\}$ such that $x_{n} \rightarrow 1^{-}$then cond $(\mathrm{F}(T))=\{0\}$ and $T$ is conditionally biased quasi-nonexpansive (c.b.q.n.) w.r.t. the sequence $\left\{x_{n}\right\}$ in $X$.

Remark 2.4. Example 2.2 above also shows that cond $(\mathrm{F}(T))$ is a closed set even though $T$ is discontinuous at $p=2$.

We need the following lemmas to prove our main theorem:

Lemma 2.3. Let $T$ be locally quasinonexpansive at $p \in F(T)$ w.r.t. $\left\{x_{n}\right\}$ and $\lim d\left(x_{n}, F(T)\right)=0$.

Then $\left\{x_{n}\right\}$ is a Cauchy sequence.
Proof. Since $\lim _{n \rightarrow \infty} d\left(x_{n}, F(T)\right)=0$ then for any given $\varepsilon>0$ there exists $n_{1} \in \square$ such that for each $n \geq n_{1}$, $d\left(x_{n}, F(T)\right)<\frac{\varepsilon}{2}$ So, there exists $q \in F(T)$ such that for all $n \geq n_{1}, d\left(x_{n}, q\right)<\frac{\varepsilon}{2}$.

Thus, for any $m, n \geq n_{1}$ we have $d\left(x_{m}, x_{n}\right) \leq d\left(x_{m}, q\right)+d\left(x_{n}, q\right)<\frac{\varepsilon}{2}+\frac{\varepsilon}{2}=\varepsilon, q \in F(T)$,

Hence $\left\{x_{n}\right\}$ is a Cauchy sequence.

Lemma 2.4. Let $T$ be conditionally biased quasinonexpansive w.r.t. $\left\{x_{n}\right\}$, and $\lim _{n \rightarrow \infty} \inf d\left(x_{n}, F(T)\right)=0$ Then:

1) $\left\{x_{n}\right\}$ converges to a point $p$ in $\operatorname{cond}(F(T))$ and $T$ is locally quasi-nonexpansive at $p \in \operatorname{cond}(\mathrm{F}(T))$ w.r.t. $\left\{x_{n}\right\}$.

2) $\left\{x_{n}\right\}$ is a Cauchy sequence.

Proof. 1) Since $T$ is conditionally biased quasinonexpansive w.r.t. $\left\{x_{n}\right\}$, it follows that $\operatorname{cond}(F(T))$ $\neq \varnothing$. As $\lim _{n \rightarrow \infty} \inf d\left(x_{n}, F(T)\right)=0$ we have that $\limsup _{n \rightarrow \infty} d\left(x_{n}, p\right)=0$ for some $p \in \operatorname{cond}(\mathrm{F}(T))$.So, we have $\lim _{n \rightarrow \infty} d\left(x_{n}, p\right)=0$ for some $p \in \operatorname{cond}(\mathrm{F}(T))$; i.e., $\left\{x_{n}\right\}$ converges to a point $p$ in $\operatorname{cond}(\mathrm{F}(T))$ and $T$ is locally quasi-nonexpansive at $p \in \operatorname{cond}(\mathrm{F}(T))$ w.r.t. $\left\{x_{n}\right\}$.

2) From $\lim _{n \rightarrow \infty} d\left(x_{n}, p\right)=0$ it follows that for any given $\varepsilon>0$ there exists $n_{1} \in \mathbf{N}$ such that for each $n \geq n_{1}, d\left(x_{n}, p\right)<\frac{\varepsilon}{2}$. Thus, for any $m, n \geq n_{1}$, we have $d\left(x_{m}, x_{n}\right) \leq d\left(x_{m}, q\right)+d\left(x_{n}, q\right)<\frac{\varepsilon}{2}+\frac{\varepsilon}{2}=\varepsilon \quad q \in F(T)$,

Hence $\left\{x_{n}\right\}$ is a Cauchy sequence.

The following lemma follows easily.

Lemma 2.5. Let $T$ be biased quasi-nonexpansive w.r.t. $\left\{x_{n}\right\}$, and let $\left\{x_{n}\right\}$ converges to a point $p$ in $F(\mathrm{~T})$ Then:

1) $\left\{x_{n}\right\}$ converges to a point $p$ in $\operatorname{cond}(F(T))$ and $T$ is conditionally biased quasi-nonexpansive w.r.t. $\left\{x_{n}\right\}$;

2) $\left\{x_{n}\right\}$ is a Cauchy sequence.

We now state our main theorem in the present paper.

Theorem 2.1. Let $F(T)$ be a nonempty closed set. Then

1) $\lim _{n \rightarrow \infty} d\left(x_{n}, F(T)\right)=0$ if $\left\{x_{n}\right\}$ converges to a point $p$ in $F(T)$;

2) $\left\{x_{n}\right\}$ converges to a point in $F(T)$ if 
$\lim _{n \rightarrow \infty} d\left(x_{n}, F(T)\right)=0, T$ is locally quasi-nonexpansive at $p \in F(T)$ w.r.t. $\left\{x_{n}\right\}$ and $X$ is complete.

Proof. 1) Since $F(T)$ is closed, $p \in \mathrm{F}(T)$ and the mapping $x \mapsto d(x, F(T))$ is continuous (see [1, p. 13]), then

$$
\lim _{n \rightarrow \infty} d\left(x_{n}, F(T)\right)=d\left(\lim _{n \rightarrow \infty} x_{n}, F(T)\right)=d(p, F(T))=0
$$

2) From Lemma 2.3, $\left\{x_{n}\right\}$ is a Cauchy sequence. Since $X$ is complete, then $\left\{x_{n}\right\}$ converges to a point, say $q$ in $X$. Since $F(T)$ is closed, then

$$
0=\lim _{n \rightarrow \infty} d\left(x_{n}, F(T)\right)=d\left(\lim _{n \rightarrow \infty} x_{n}, F(T)\right)=d(p, F(T))
$$

implies that $q \in \mathrm{F}(T)$.

As consequences of Theorem 2.1, we have the following:

Corollary 2.1. Let $F(T)$ a nonempty closed set and for a given $x_{0} \in D$ and each $n \in \omega,\left\{T^{n} x_{0}\right\} \in D$ Then

1) $\lim _{n \rightarrow \infty} d\left(T^{n} x_{0}, F(T)\right)=0$ if $\left\{T^{n} x_{0}\right\}$ converges to a point $p$ in $F(T)$;

2) $\left\{T^{n} x_{0}\right\}$ converges to a point in $F(T)$ if, $\lim _{n \rightarrow \infty} d\left(T^{n} x_{0}, F(T)\right)=0, T$ is locally qusi-nonexpansive at $p \in \mathrm{F}(T)$ w.r.t. $\left\{T^{n} x_{0}\right\}$ and $X$ is complete.

Corollary 2.2. Let $X$ be a normed linear space, $F(T)$ a nonempty closed set and for a given $x_{0} \in D$ and each $n \in \omega,\left\{T_{\lambda}^{n} x_{0}\right\} \in D$.

(1) If the sequence $\left\{T_{\lambda}^{n} x_{0}\right\}$ converges to a point $p$ in $F(T)$, then

$$
\lim _{n \rightarrow \infty} d\left(T_{\lambda}^{n} x_{0}, F(T)\right)=0
$$

(2) If $\lim _{n \rightarrow \infty} d\left(T_{\lambda}^{n} x_{0}, F(T)\right)=0 \quad T$ is locally quasinonexpansive at $p \in \mathrm{F}(T)$ w.r.t. $\left\{T_{\lambda}^{n} x_{0}\right\}$ and $X$ is complete, then $\left\{T_{\lambda}^{n} x_{0}\right\}$ converges to a point $p$ in $F(T)$.

Corollary 2.3. Let $X$ be a normed linear space, $F(T)$ a nonempty closed set and for a given $x_{0} \in D$ and each $n \in \omega,\left\{T_{\lambda, \mu}^{n} x_{0}\right\} \in D$ Then

(1) $\lim _{n \rightarrow \infty} d\left(T_{\lambda, \mu}^{n} x_{0}, F(T)\right)=0$ if the sequence $\left\{T_{\lambda, \mu}^{n} x_{0}\right\}$ converges to a point $p$ in $F(T)$;

(2) $\left\{T_{\lambda, \mu}^{n} x_{0}\right\}$ converges to a point $p$ in $F(T)$ if $\lim _{n \rightarrow \infty} d\left(T_{\lambda, \mu}^{n} x_{0}, F(T)\right)=0, T$ is locally quasi-nonexpansive at $p \in \mathrm{F}(T)$ w.r.t. $\left\{T_{\lambda, \mu}^{n} x_{0}\right\}$ and $X$ is complete.

Note that the continuity of $T$ implies that $F(T)$ is closed but the converse need not be true. To effect this consider the following example.

Example 2.3. Let $X=[0, \infty)$ and $D=[0,1)$ be endowed with the Euclidean metric d. Define the map- ing $T: D \rightarrow X$ by $T(x)=x$ if $x \in\left[0, \frac{1}{2}\right]$ and $T(x)$ $=3 x^{2}$ if $x \in\left(\frac{1}{2}, 1\right)$ Obviously, $F(T)=[0,1 / 2]$ is a nonempty closed but $T$ is not continuous at $x=1 / 2$.

Remark 2.5. (a) In order to support the above fact Ahmed and Zeyada [1] stated wrongly in their Example 2.2, where $X=[0,1), D=[0,1 / 4) \cup(1 / 2,5 / 6], T(x)=x$.

If $X \in[0,1 / 4)$ and $T(x)=x / 2$ if $x \in(1 / 2,5 / 6)$ that $T$ is not continuous. In fact, we observe that in this example $T$ is continuous.

(b) From Lemma 2.1, Examples 2.1 and 2.3, the continuity of $T$ implies that $F(T)$ is closed but the converse may not be true; then we have that Corollaries 2.1, 2.2 and 2.3 are improvement of Theorem 1.1 in [13, p.462], Theorem 1.1' in [13, p. 469], and Theorem 3.1 in [8, p. 98], respectively.

(c) Since every quasi-nonexpansive map w.r.t. a sequence $\left\{x_{n}\right\}$ is locally quasi-nonexpansive at each $p \in F(T)$ w.r.t. a sequence $\left\{x_{n}\right\}$, but the converse may not be true; we have that Theorem 2.1, Corollaries 2.1, 2.2 and 2.3 are improvement of corresponding Theorem 2.1, Corollary 2.1, 2.2 and 2.3 of Ahmed and Zeyada [1].

(d) By considering the closedness of $F(T)$ in lieu of the continuity of $T$ and $T: D \rightarrow X$ instead of $T: X \rightarrow X$ we have that our Corollary 2.1 improves Proposition 1.1 of Kirk [10, p. 168].

(e) The closedness condition of $D$ in Theorem 1.1 and $1.1^{\prime}$ of Petryshyn and Williamson [12, p. 462, 469] and Theorem 3.1 in [8, p. 98] is superfluous.

(f) The convexity condition of $D$ in Theorem $1.1^{\prime}$ of Petryshyn and Williamson [12, p. 469] is superfluous because the author assumed in their theorem that $\left\{T_{\lambda}^{n} x_{0}\right\} \in D$ for each $n \in \omega$ and a given $x_{0} \in D$ in condition $\left(1.3^{\prime}\right)$.

Theorem 2.2. Let cond $(F(T))$ be a nonempty closed set. Then $\left\{x_{n}\right\}$ converges to a point in cond $(F(T))$ if $\liminf _{n \rightarrow \infty} d\left(x_{n}\right.$, cond $\left.(F(T))\right)=0, T$ is condionally biased quasi-nonexpansive w.r.t. $\left\{x_{n}\right\}$ and $X$ is complete.

Proof. Since cond $(F(T)) \subset F(T)$ we have that $\liminf _{n \rightarrow \infty} d\left(x_{n}\right.$, cond $\left.(F(T))\right)=0$ implies $\liminf _{n \rightarrow \infty} d\left(x_{n}\right.$, $F(T))=0$ Now using the technique of the proof of Theorem 2.1 the conclusion follows from Lemma 2.3.

The following results follows easily from Lemma 2.5 .

Theorem 2.3. Let $F(T)$ be a nonempty closed set. Then $\left\{x_{n}\right\}$ converges to a point in $\operatorname{cond}(F(T))$ if $\left\{x_{n}\right\}$ converges to a point $p$ in $F(T), T$ is biased quasi-nonexpansive w.r.t. $\left\{x_{n}\right\}$ and $X$ is complete. 
Theorem 2.4. Let $X$ be a complete metric space and let $\operatorname{cond}(F(T))$ be a nonempty closed set. Assume that

1) $T$ is biased quasi-nonexpansive w.r.t. $\left\{x_{n}\right\}$;

2) $\lim _{n \rightarrow \infty} d\left(x_{n}, x_{n+1}\right)=0$ or $\left\{x_{n}\right\}$ is a Cauchy sequence;

3) if the sequence $\left\{y_{n}\right\}$ satisfies $\lim _{n \rightarrow \infty} d\left(y_{n}, y_{n+1}\right)=0$ then

or

$$
\liminf _{n \rightarrow \infty} d\left(y_{n}, \operatorname{cond}(F(T))\right)=0
$$

$$
\limsup _{n \rightarrow \infty} d\left(y_{n}, \operatorname{cond}(F(T))\right)=0 .
$$

Then $\left\{x_{n}\right\}$ converges to a point in $\operatorname{cond}(F(T))$.

Proof. Since $\operatorname{cond}(F(T)) \neq \varnothing$ it follows from (i) that $T$ is condionally biased quasi-nonexpansive w.r.t. $\left\{x_{n}\right\}$ and the sequence $\left\{d\left(x_{n}, \operatorname{cond}(F(T))\right)\right\}$ is monotonically decreasing and bounded from below by zero.

Then $\liminf _{n \rightarrow \infty} d\left(x_{n}\right.$, cond $\left.(F(T))\right)$ exists.

From 2) and 3), we have that

$$
\lim _{n \rightarrow \infty} \inf d\left(x_{n}, \operatorname{cond}(F(T))\right)=0
$$

or

$$
\lim _{n \rightarrow \infty} \sup d\left(x_{n}, \operatorname{cond}(F(T))\right)=0 .
$$

Then $\lim _{n \rightarrow \infty} d\left(x_{n}\right.$, cond $\left.(F(T))\right)=0$ Therefore, by Theorem 2.2, the sequence $\left\{x_{n}\right\}$ converges to a point in $\operatorname{cond}(F(T))$.

As consequences of Theorem 2.4, we obtain the following:

Corollary 2.4. Let $X$ be a complete metric space and let $\operatorname{cond}(F(T))$ be a nonempty closed set. Assume that

1) $T$ is biased quasi-nonexpansive w.r.t. $\left\{x_{n}\right\}$;

2) $T$ is asymptoticc regular at $x_{0} \in D$ ( or $\left\{T^{n}\left(x_{0}\right)\right\}$ is a Cauchy sequence );

$3)$ if the sequence $\left\{y_{n}\right\}$ satisfies $\lim _{n \rightarrow \infty} d\left(y_{n}, y_{n+1}\right)=0$ then

$$
\liminf _{n \rightarrow \infty} d\left(y_{n}, \operatorname{cond}(F(T))\right)=0
$$

or

$$
\lim _{n \rightarrow \infty} \sup d\left(y_{n}, \operatorname{cond}(F(T))\right)=0 .
$$

Then $\left\{T^{n}\left(x_{0}\right)\right\}$ converges to a point in $\operatorname{cond}(F(T))$.

Corollary 2.5. Let $X$ be a Banach space and let $\operatorname{cond}(F(T))$ be a nonempty closed set. Assume that
1) $T$ is biased quasi-nonexpansive w.r.t. $\left\{T^{n}\left(x_{0}\right)\right\}$;

2) $T$ is asymptoticc regular at $x_{0} \in D$ (or $\left\{T^{n}\left(x_{0}\right)\right\}$ is a Cauchy sequence );

3) if the sequence $\left\{y_{n}\right\}$ satisfies $\lim _{n \rightarrow \infty}\left\|y_{n}-T_{\lambda} y_{n}\right\|=0$, then

$$
\liminf _{n \rightarrow \infty} d\left(y_{n}, \operatorname{cond}(F(T))\right)=0
$$

or

$$
\lim _{n \rightarrow \infty} \sup d\left(y_{n}, \operatorname{cond}(F(T))\right)=0 .
$$

Then $\left\{T^{n}\left(x_{0}\right)\right\}$ converges to a point in $\operatorname{cond}(F(T))$. Corollary 2.6. Let $X$ be a Banach space and let $\operatorname{cond}(F(T))$ be a nonempty closed set. Assume that

1) $T$ is biased quasi-nonexpansive w.r.t. $\left\{T_{\lambda, \mu}^{n}\left(x_{0}\right)\right\}$;

2) $T$ is asymptoticc regular at $x_{0} \in D$ (or $\left\{T_{\lambda, \mu}^{n}\left(x_{0}\right)\right\}$ is a Cauchy sequence );

3) if the sequence $\left\{y_{n}\right\}$ satisfies $\lim _{n \rightarrow \infty}\left\|y_{n}-T_{\lambda, \mu} y_{n}\right\|=0$, then

$$
\liminf _{n \rightarrow \infty} d\left(y_{n}, \operatorname{cond}(F(T))\right)=0
$$

or

$$
\lim _{n \rightarrow \infty} \sup d\left(y_{n}, \operatorname{cond}(F(T))\right)=0 .
$$

Then $\left\{T_{\lambda, \mu}^{n}\left(x_{0}\right)\right\}$ converges to a point in $\operatorname{cond}(F(T))$.

Remark 2.6. From Lemmas 2.1 and 2.2, Examples 2.1 and 2.3, Remark 2.3, the continuity of $T$ implies that $F(T)$ is closed but the converse may not be true; we obtain that Corollary 2.4 include Theorem 1.2 in [12, p. 464] and Theorem 3.2 in [7, p. 99] as special cases.

As another consequence of Theorem 2.1, we establish the following theorem:

Theorem 2.5. Let $X$ be a complete metric space and let cond $(F(T))$ be a nonempty closed set. Assume that

1) $T$ is biased quasi-nonexpansive w.r.t. $\left\{x_{n}\right\}$;

2) for every $x \in D-\operatorname{cond}(F(T))$ there exists $p_{x} \in$ cond $(F(T))$ such that $d\left(x_{n+1}, p_{x}\right)<d\left(x_{n}, p_{x}\right)$;

3) the sequence $\left\{x_{n}\right\}$ contains a subsequence $\left\{x_{n_{j}}\right\}$ converging to $x^{*} \in D$.

Then $\left\{x_{n}\right\}$ converges to a point in $\operatorname{cond}(F(T))$.

Proof. Since $\operatorname{cond}(F(T)) \neq \varnothing$ it follows from (i) that $T$ is condionally biased quasi-nonexpansive w.r.t. $\left\{x_{n}\right\}$ and the sequence $\left\{d\left(x_{n}\right.\right.$, cond $\left.\left.(F(T))\right)\right\}$ is monotonically decreasing and bounded from below by zero. Then $\lim _{n \rightarrow \infty} d\left(x_{n}\right.$, cond $\left.(F(T))\right)=d\left(\lim _{n \rightarrow \infty} x_{n}\right.$, $\left.\operatorname{cond}(F(T))\right)$ 
$=r \geq 0$ exists. We now apply Theorem 2.4. It suffices to show that $\mathrm{r}=0$. If $\lim _{n \rightarrow \infty} x_{n}=x^{*} \in \operatorname{cond}(F(T))$ then $r=0$. If $x^{*} \notin \operatorname{cond}(F(T))$ then $x^{*} \in D-\operatorname{cond}(F(T))$ Thus there exists $p_{x^{*}} \in \operatorname{cond}(F(T))$ such that

$$
\begin{aligned}
d\left(x^{*}, p_{x^{*}}\right) & =d\left(\lim _{n \rightarrow \infty} x_{n+1}, p_{x^{*}}\right)=\lim _{n \rightarrow \infty} d\left(x_{n+1}, p_{x^{*}}\right) \\
& <\lim _{n \rightarrow \infty} d\left(x^{*}, p_{x^{*}}\right)=d\left(\lim _{n \rightarrow \infty} x_{n}, p_{x^{*}}\right)=d\left(x^{*}, p_{x^{*}}\right)
\end{aligned}
$$

This is a contradiction. So, $x^{*} \in \operatorname{cond}(F(T))$.

Corollary 2.7. Let $X$ be a complete metric space, cond $(F(T))$ a nonempty closed set and for a given $x_{0} \in D$ and each $n \in \omega,\left\{T^{n} x_{0}\right\} \in D$. Assume that

1) $T$ is biased quasi-nonexpansive w.r.t. $\left\{T^{n}\left(x_{0}\right)\right\}$;

2) for every $x \in D-\operatorname{cond}(F(T))$ there exists $p_{x} \in \operatorname{cond}(F(T))$ such that

$$
d\left(T^{n+1}\left(x_{0}\right), p_{x}\right)<d\left(T^{n}\left(x_{0}\right), p_{x}\right) ;
$$

3) the sequence $\left\{T^{n}\left(x_{0}\right)\right\}$ contains a subsequence $\left\{T^{n_{j}}\left(x_{0}\right)\right\}$ converging to $x^{*} \in D$.

Then $\left\{T^{n}\left(x_{0}\right)\right\}$ converges to a point in cond $(F(T))$.

Corollary 2.8. Let $X$ be a Banach space, cond $(F(T))$ a nonempty closed set and for a given $x_{0} \in D$ and each $n \in \omega,\left\{T_{\lambda, \mu}^{n}\left(x_{0}\right)\right\} \in D$ Assume that

1) $T$ is biased quasi-nonexpansive w.r.t. $\left\{T_{\lambda, \mu}^{n}\left(x_{0}\right)\right\}$;

2) for every $x \in D-\operatorname{cond}(F(T))$ there exists $p_{x} \in$ cond $(F(T))$ such that

$$
\left\|T_{\lambda}^{n+1}\left(x_{0}\right)-p_{x}\right\|<\left\|T_{\lambda}^{n}\left(x_{0}\right)-p_{x}\right\| ;
$$

3) the sequence $\left\{T^{n}\left(x_{0}\right)\right\}$ contains a subsequence $\left\{T_{\lambda}^{n_{j}}\left(x_{0}\right)\right\}$ converging to $x^{*} \in D$.

Then $\left\{T_{\lambda}^{n_{j}}\left(x_{0}\right)\right\}$ converges to a point in cond $(F(T))$.

Corollary 2.9. Let $X$ be a Banach space, cond $(F(T))$ a nonempty closed set and for a given $x_{0} \in D$ and each $n \in \omega,\left\{T_{\lambda, \mu}^{n}\left(x_{0}\right)\right\} \in D$ Assume that

1) $T$ is biased quasi-nonexpansive w.r.t. $\left\{T_{\lambda, \mu}^{n}\left(x_{0}\right)\right\}$;

2) for every $x \in D-\operatorname{cond}(F(T))$ there exists $p_{x} \in \operatorname{cond}(F(T))$ such that

$$
\left\|T_{\lambda, \mu}^{n+1}\left(x_{0}\right)-p_{x}\right\|<\left\|T_{\lambda, \mu}^{n}\left(x_{0}\right)-p_{x}\right\| ;
$$

3) the sequence $\left\{T^{n}\left(x_{0}\right)\right\}$ contains a subsequence
$\left\{T_{\lambda, \mu}^{n_{j}}\left(x_{0}\right)\right\}$ converging to $x^{*} \in D$.

Then $\left\{T_{\lambda, \mu}^{n}\left(x_{0}\right)\right\}$ converges to a point in cond $(F(T))$.

Remark 2.7. From Lemmas 2.1 and 2.2, Examples 2.1 and 2.3, Remark 2.3, the continuity of $T$ implies that $F(T)$ is closed but the converse may not be true ; we obtain that Corollary 2.7 is an improvement of Theorem 1.3 in [13, p. 466].

\section{Applications to Geometry of Banach Spaces}

Throughout this section, let $\mathbf{R}$ denote the set of real numbers. Let $K=K(z, r)$ be a closed ball in a Banach space $X$. For a sequence $\left\{x_{n}\right\}_{n=0}^{\infty}$ Ú $K$ converging to $X$ we define

$$
\lim _{n \rightarrow \infty} \mathrm{D}_{n}=\operatorname{SD}(x, K)
$$

where

$$
\mathrm{D}_{0}=\operatorname{conv}\left(\left\{x_{0}\right\} \cup K\right)
$$

and

$$
\mathrm{D}_{n+1}=\operatorname{conv}\left(\left\{x_{n}\right\} \cup \mathrm{D}_{n}\right) \forall n \in \omega
$$

and $\operatorname{SD}(x, K)$ is called a super drop .

Clearly, for a constant sequence $\left\{x_{n}\right\} \equiv\{x\}$ converging to $x$ we have $\mathrm{D}_{n+1}=\mathrm{D}_{n} \forall n \in \omega$ so that $\mathrm{D}(x, k)=\operatorname{conv}(\{x\} \cup K)$ and is called a drop Thus the concept of a drop is a special case of super drop It is also clear that if $y \in \mathrm{D}(x, K)$ then $\mathrm{D}(y, K) \subset \mathrm{D}(x, K)$ and if $z=0$ then $\|y\|=\|x\|$.

Recall that a function $\varphi: X \rightarrow \mathbf{R}$ is called a lower semicontinuous whenever $\{x \in X: \varphi(x) \leq a\}$ is closed for each $a \in \mathbf{R}$.

Caristi [4] proved the following:

Theorem A. Let $(X, d)$ be complete and $\varphi: X \rightarrow \mathbf{R}$ a lower semicontinuous function with a finite lower bound. Let $T: X \rightarrow X$ be any function such that $d(x, T(x)) \leq \varphi(x)-\varphi(T(x))$ for each $x \in X$. Then $T$ has a fixed point.

We now state and prove some applications of our main results in section 2 to geometry of Banach Spaces.

Theorem 3.1. Let $C$ be a closed subset of a Banach space $X$ let $z \in X-C$ and let $K=K(z, r)$ be a closed ball of radius $r<d(z, C)=R$ Let $x$ be an arbitrary element of $C$ let $\left\{x_{n}\right\}$ be a sequence in $C$ converging to $X$ and let $T: C \rightarrow X$ be any continuous function defined implicitly by $T(x) \in C \cap \operatorname{SD}(x, K)$ for each $x \in C$ in the sense that $T\left(x_{n}\right) \in C \cap \mathrm{D}_{n}$ for each $n \in \omega$. Then 
1) $\lim _{n \rightarrow \infty} d\left(x_{n}, F(T)\right)=0$ if $\left\{x_{n}\right\}$ converges to a point $p$ in $F(T)$;

2) $\left\{x_{n}\right\}$ converges to a point in $F(T)$ if $\lim _{n \rightarrow \infty} d\left(x_{n}, F(T)\right)=0, T$ is locally quasi-nonexpansive at $p \in F(T)$ w.r.t. $\left\{x_{n}\right\}$.

Proof. Without loss of generality we may assume that $z=0$. Let $\|x\|=\eta \geq R$ and let $X=A \cap \operatorname{SD}(x, K)$ Then it is clear that $T$ maps $X$ into itself. For given $y \in X$ and a sequence $\left\{y_{n}\right\}$ converging to $y$, we shall estimate $\|y-T(y)\|$ on $X$.

For given $y \in X$ and the corresponding sequence $\left\{y_{n}\right\}$ there is a sequence $\left\{b_{n}\right\}$ in $X$ with $T\left(y_{n}\right)=t b_{n}+(1-t) y_{n}, 0<t<1 \quad$ Now $\left\|T\left(y_{n}\right)\right\| \leq t\left\|b_{n}\right\|$ $+(1-t)\left\|y_{n}\right\|$, we have

$$
t\left(\left\|y_{n}\right\|-\left\|b_{n}\right\|\right) \leq\left\|y_{n}\right\|-\left\|T\left(y_{n}\right)\right\|
$$

so because $\left\|y_{n}\right\|-\left\|b_{n}\right\| \geq R-\eta$, we find that

$$
t \leq \frac{\left\|y_{n}\right\|-\left\|T\left(y_{n}\right)\right\|}{R-\eta} .
$$

Thus,

$$
\begin{aligned}
\left\|y_{n}\right\|-\left\|T\left(y_{n}\right)\right\| & \leq t\left\|y_{n}-b_{n}\right\| \\
& \leq t\left(\left\|y_{n}\right\|+\left\|b_{n}\right\|\right) \leq(\eta+r) \\
& \leq \frac{\eta+r}{R-r}\left(\left\|y_{n}\right\|-\left\|T\left(y_{n}\right)\right\|\right)
\end{aligned}
$$

Define $d(x, y)=\|x-y\| \forall x, y \in X$ and $\varphi(y)$ $=\frac{\eta+r}{R-r}\|y\|$ then $X$ is complete as a metric space and $\varphi: X \rightarrow \mathbf{R}$ is a continuous function. So, $\varphi$ is a lower-semicontinuous function. Also, the above inequality takes the form $d\left(y_{n}, T\left(y_{n}\right)\right) \leq \varphi\left(y_{n}\right)-\varphi\left(T\left(y_{n}\right)\right)$. Proceeding to the limit as $n \rightarrow \infty$ we obtain $d(y, T(y)) \leq \varphi(y)-\varphi(T(y))$ for each $y \in X$. There- fore, applying the theorem of Caristi we obtain that $T$ has a fixed point $p=p(x)$ for each $x \in C$, i.e., $F(T) \neq \varnothing$. By continuity of $T, F(T)$ is closed. Hence the conclusion follows from Theorem 2.1.

Since drop is a special case of super drop, we have the following:

Corollary 3.1. Let $C$ be a closed subset of a Banach space $X$ let $z \in X-C$ and let $K=K(z, r)$ be a closed ball of radius $r<d(z, C)=R$ Let $x$ be an arbitrary element of $C$, and let $T: C \rightarrow X$ be any (not necessarily continuous) function defined implicitly by $T(x) \in C \cap \mathrm{D}(x, K)$ for each $x \in C$. Then

(1) $\lim _{n \rightarrow \infty} d\left(x_{n}, F(T)\right)=0$ if $\left\{x_{n}\right\}$ converges to a point $p$ in $F(T)$;
(2) $\left\{x_{n}\right\}$ converges to a point in $F(T)$ if $\lim _{n \rightarrow \infty} d\left(x_{n}, F(T)\right)=0, T$ is locally quasi-nonexpansive at $p \in F(T)$ w.r.t. $\left\{x_{n}\right\}$.

We now prove the following result for biased quasinonexpansive mapping w.r.t. a sequence $\left\{x_{n}\right\}$.

Theorem 3.2. Let $C$ be a closed subset of a Banach space $X$ let $z \in X-C$ and let $K=K(z, r)$ be a closed ball of radius $r<d(z, C)=R$ Let $x$ be an arbitrary element of $C,\left\{x_{n}\right\}$ a sequence in $C$ converging to $X$, and let $T: C \rightarrow X$ be any con- tinuous function defined implicitly by $T(x) \in C \cap \mathrm{SD}(x, K)$ for each $x \in C$ in the sense that $T\left(x_{n}\right) \in C \cap \mathrm{D}_{n}$ for each $n \in \omega$. If $\left\{x_{n}\right\}$ converges to a point in $F(T), T$ is biased quasi-nonexpansive w.r.t. $\left\{x_{n}\right\}$ then $\left\{x_{n}\right\}$ converges to a point in $\operatorname{cond}(F(T))$.

Proof. Using Theorem 2.3. instead of Theorem 2.1 the conclusion follows on the lines of the proof technique of Theorem 3.1.

As a consequence of Theorem 3.2, we obtain the following:

Corollary 3.2. Let $C$ be a closed subset of a Banach space $X$ let $z \in X-C$ and let $K=K(z, r)$ be a closed ball of radius $r<d(z, C)=R$. Let $x$ be an arbitrary element of $C$, and let $T: C \rightarrow X$ be any (not necessarily continuous) function defined implicitly by $T(x) \in C \cap \mathrm{D}(x, K)$ for each $x \in C$. If $\left\{x_{n}\right\}$ converges to a point in $F(T), T$ is biased quasi-nonexpansive w.r.t. $\left\{x_{n}\right\}$ then $\left\{x_{n}\right\}$ converges to a point in cond $(F(T))$.

Open Question. To what extent can the continuity hypothesis on $T$ be muted in Theorems 3.1 and 3.2?

\section{References}

[1] M. A. Ahmed and F. M. Zeyad, "On Convergence of a Sequence in Complete Metric Spaces and its Applications to Some Iterates of Quasi-Nonexpansive Mappings," Journal of Mathematical Analysis and Applications, Vol. 274, No. 1, 2002, pp. 458-465. doi:10.1016/S0022-247X(02)00242-1

[2] J.-P. Aubin, “Applied Abstract Analysis,” Wiley-Interscience, New York, 1977.

[3] F. E. Browder and W. V. Petryshyn, “The Solution by Iteration of Nonlinear Functional Equations in Banach Spaces," Bulletin of the American Mathematical Society, Vol. 272, 1966, pp. 571-575. doi:10.1090/S0002-9904-1966-11544-6

[4] J. Caristi, "Fixed Point Theorems for Mappings Satisfying Inwardness Conditions," Transaction of the American Mathematical Society, Vol. 215, 1976, pp. 241-251. doi:10.1090/S0002-9947-1976-0394329-4

[5] J. B. Diaz and F. T. Metcalf, "On the Set of Sequencial Limit Points of Successive Approximations," Transactions of the American Mathematical Society, Vol. 135, 
1969, pp. 459-485.

[6] W. G. Dotson Jr., "On the Mann Iteration Process," Transaction of the American Mathematical Society, Vol. 149, 1970, pp. 65-73.

doi:10.1090/S0002-9947-1970-0257828-6

[7] W. G. Dotson Jr., "Fixed Points of Quasinon-Expansive Mappings," Journal of the Australian Mathematical Society, Vol. 13, 1972, pp. 167-170.

[8] M. K. Ghosh and L. Debnath, "Convergence of Ishikawa Iterates of Quasi-Nonexpansive Mappings,” Journal of Mathematical Analysis and Applications, Vol. 207, No. 1, 1997, pp. 96-103. doi:10.1006/jmaa.1997.5268

[9] S. Ishikawa, "Fixed Points by a New Iteration Method," Proceedings of the American Mathematical Society, Vol. 44, No. 1, 1974, pp. 147-150. doi:10.1090/S0002-9939-1974-0336469-5
[10] W. A. Kirk, "Remarks on Approximationand Approximate Fixed Points in Metric Fixed Point Theory," $A n$ nales Universitatis Mariae Curie-Sklodowska, Section A, Vol. 51, No. 2, 1997, pp. 167-178.

[11] W. A. Kirk, "Nonexpansive Mappings And Asymptotic Regularity," Ser. A: Theory Methods, Nonlinear Analysis, Vol. 40, No. 1-8, 2000, pp. 323-332.

[12] W. R. Mann, “Mean Valued Methods In Iteration,” Proceedings of the American Mathematical Society, Vol. 4, No. 3, 1953, pp. 506-510. doi:10.1090/S0002-9939-1953-0054846-3

[13] W. V. Petyshyn and T. E. Williamson Jr., "Strong and Weak Convergence of The Sequence of Successive Approximations for Quasi-Nonexpansive Mappings,” Journal of Mathematical Analysis and Applications, Vol. 43, 1973, pp. 459-497.

doi:10.1016/0022-247X(73)90087-5 